\title{
AN INTEGRABLE FOURTH-ORDER NONLINEAR EVOLUTION EQUATION APPLIED TO SURFACE REDISTRIBUTION DUE TO CAPILLARITY
}

\author{
PETER TRITSCHER ${ }^{\prime}$
}

(Received 27 August 1995; revised 13 September 1995)

\begin{abstract}
Members of an hierarchy of integrable nonlinear evolution equations, related to the wellknown linearizable diffusion equation which has the diffusivity form as the reciprocal of the square of the concentration, are adapted to derive a new integrable nonlinear equation which models the surface evolution of an arbitrarily-oriented theoretical anisotropic material by the concomitant action of evaporation-condensation and surface diffusion. The constitutive relations are explicitly formulated and these show that the theoretical anisotropic material behaves like a liquid crystal. The integrable nonlinear equation may be used to advantage as test cases for numerical schemes. Its form has many attributes of the nonlinear governing equation for an isotropic material. Closed-form solutions are constructed for the evolution of a ramped surface by concomitant evaporation-condensation and surface diffusion.
\end{abstract}

\section{Introduction}

Fundamental to the study of interfaces are the kinetics of morphological changes due to capillarity. Although capillary driving forces appear rather insignificant, on a sufficiently small scale they become dominant and play an important role in such varied phenomena as the sintering of crystalline powders [21], coarsening and spheroidization of precipitate particles in alloys [16], the growth of grain boundary voids during creep [61], the formation of "island" structures in thin films [57, 70], blunting of field-emission cathodes [56], faceting of high temperature catalysts [49], the formation of cystallite catalysts [73] and the development of grooves when grain boundaries meet a surface $[1,50]$. For each of these, the interface will tend to assume a configuration so as to reduce the surface energy to a minimum. For most solid materials, and particularly crystalline materials, the surface tension, denoted $\gamma$, varies with orientation. If this variation is sufficiently marked, the equilibrium shape of

\footnotetext{
${ }^{1}$ Department of Mathematics, University of Wollongong, 2522, Australia

(C) Australian Mathematical Society, 1997, Serial-fee code 0334-2700/97
} 
crystals will be polyhedral and planar surfaces of certain orientations may be unstable with respect to the spontaneous decomposition into a surface composed of segments of two or more orientations, even though this process involves a net increase in the total surface area [2].

Relaxation of the surface may occur by a number of mechanisms, transport by surface diffusion along the interface; transport by volume or bulk diffusion either by defects in the solid or by solution in the fluid phase; transport by evaporationcondensation in a single-component system and transport by plastic or viscous flow [46, 32].

Kuczynski [46] carried out pioneering work on sintering and determined growth laws for the interface radius for each transport mechanism in the sintering of spheres to a planar surface and deduced that surface diffusion is dominant for small particle size. Further innovative work was carried out by Herring $[33,34]$ who outlined a general procedure for calculating morphological change induced by capillarity and explicitly related chemical potential and curvature for anisotropic $\gamma$. For materials with isotropic properties, Mullins, co-workers and others $[29,37,40,52,55]$ have derived general solutions for the Cauchy problem of describing the relaxation of a nearly-planar surface for each of the transport processes. The problems were linearized by assuming the slope was everywhere small and invariant forms were calculated that characterize the profile for a point source, an antisymmetric and a symmetric scratch [55].

Brailsford and Gjostein [8] investigate the influence of surface-energy anisotropy on morphological changes occurring by surface diffusion on simply-shaped bodies. Approximate solutions describing the relaxation, from an assumed initial circle to the final equilibrium shape are derived. Also, they determine the form of $\gamma$ for stability. They discuss three spatial dimensions. Furthering work on anisotropic materials, Bonzel and Preuss [5] and Bonzel et al. [6] consider evolution of periodic profiles for a theoretical material which has cusp-like anisotropic surface tension. It is shown that relatively-planar segments develop at the cusp orientation in agreement with experiment (Preuss et al. [59]). The analysis was carried out by numerical methods. Srolovitz and Safran $[70,71]$ consider capillary instabilities in thin films and determine a critical radius below which holes contract.

Ghez [26], Davi and Gurtin [19] and Gurtin [30] have derived general balance laws for non-stationary interfaces which may be in a state of non-equilibrium. These results generalize the work of Herring $[33,34]$ which assumes near-equilibrium conditions. Taylor et al. [74] give a review of mathematical methods for the treatment of geometric models of interface motion. They compare the various methods and discuss limitations. Additionally, there is a recent review of surface motion by surface diffusion by Cahn and Taylor [15]. Geometry growth laws for morphological change are developed from both a physical model and from gradient flows. A survey of mathematical techniques is given. 
Here, we derive a new integrable nonlinear evolution equation which gives scope to compose closed-form solutions for surface relaxation for a wider range of surface slopes than presently available. Closed-form solutions are constructed for the redistribution of an extended solid surface of a single-component system by the concomitant action of evaporation-condensation and surface diffusion.

\section{Preliminaries}

Where we have only one material species present, the driving force of the evaporation-condensation and surface diffusion is due to capillary effects and to surface tension anisotropy. After Mullins [55], the motion normal to the surface, $\partial N / \partial t$, with only one non-zero principal curvature, $\kappa$, is given by

$$
\begin{aligned}
\frac{\partial N}{\partial t}= & -p_{0}(2 \pi \bar{m})^{-1 / 2}(k T)^{-3 / 2} \Omega^{2} \alpha(\theta)\left(\gamma(\theta)+\frac{d^{2} \gamma}{d \theta^{2}}\right) \kappa \\
& +\left(\Omega^{2} c_{0} / k T\right) \frac{\partial}{\partial s}\left\{D_{s}(\theta) \frac{\partial}{\partial s}\left[\left(\gamma(\theta)+\frac{d^{2} \gamma}{d \theta^{2}}\right) \kappa\right]\right\}
\end{aligned}
$$

where $p_{0}$ is the equilibrium vapor pressure over a flat surface, $\bar{m}$ is the mass of an atom, $k$ is Boltzmann's constant, $T$ is the absolute temperature, $\Omega$ is the atomic volume, $\alpha(\theta)$ is the condensation coefficient $(\leq 1)$ which may be dependent on the orientation $\theta[39,33], \gamma(\theta)$ is the surface tension dependent on the orientation, $c_{0}$ is the number of atoms per unit area in one monolayer, $D_{s}(\theta)$ is the diffusivity, also dependent on the orientation and $s$ is arclength. The first term on the right-hand side is the contribution from evaporation-condensation and the second term represents the surface diffusion component.

In Cartesian coordinates, the evolution of the surface profile, denoted $y(x, t)$, is

$$
\begin{aligned}
y_{t}= & p_{0}(2 \pi \bar{m})^{-1 / 2}(k T)^{-3 / 2} \Omega^{2} \alpha(\theta)\left(\gamma(\theta)+\frac{d^{2} \gamma}{d \theta^{2}}\right) \frac{y_{x x}}{\left(1+y_{x}^{2}\right)} \\
& -\left(\Omega^{2} c_{0} / k T\right) \frac{\partial}{\partial x}\left\{\frac{D_{s}(\theta)}{\left(1+y_{x}^{2}\right)^{1 / 2}} \frac{\partial}{\partial x}\left[\left(\gamma(\theta)+\frac{d^{2} \gamma}{d \theta^{2}}\right) \frac{y_{x x}}{\left(1+y_{x}^{2}\right)^{3 / 2}}\right]\right\},
\end{aligned}
$$

where we assume the usual convention $\kappa>0$ when $y_{x x}<0$ and we choose $\theta$ to be the angle from the $y$ axis to the normal of the surface, hence $\theta=\arctan (d y / d x)$.

For isotropic materials we have

$$
y_{t}=A \frac{y_{x x}}{\left(1+y_{x}^{2}\right)}-B \frac{\partial}{\partial x}\left\{\frac{1}{\left(1+y_{x}^{2}\right)^{1 / 2}} \frac{\partial}{\partial x}\left[\frac{y_{x x}}{\left(1+y_{x}^{2}\right)^{3 / 2}}\right]\right\},
$$

where $A=p_{0}(2 \pi \bar{m})^{-1 / 2}(k T)^{-3 / 2} \Omega^{2} \alpha_{0} \gamma_{0}$ and $B=\Omega^{2} c_{0} D_{0} \gamma_{0} / k T$ with $\alpha_{0}, \gamma_{0}, D_{0}$ constants for the condensation coefficient, surface tension and surface diffusivity respectively. 
There are no known analytic solutions for (3) (Cahn and Taylor [15], except if evaporation-condensation only is operative, $B=0$, then an explicit exact solution exists for the development of a symmetric grain boundary groove (Broadbridge [11]). Recently, some progress has been made toward describing the effect of the nonlinearity of (3). The smoothing properties when $B=0$ have been investigated rigorously by Kitada [41, 43] and by Kitada and Umehara [42]. The existence and uniqueness of solution have been proved for the evolution, due to surface diffusion, of a convex interface that appears stationary to an observer moving with uniform translational velocity (Davi and Gurtin [19]) and for the boundary value problem of the development of a symmetric grain boundary groove (Bouchiba [7]).

Most applications to boundary value problems assume the surface slope is everywhere small, $\left|y_{x}\right| \ll 1$, then use the linearized equation

$$
y_{t}=A y_{x x}-B y_{x x x x} .
$$

For example surface redistribution due to capillarity (Mullins [52], King and Mullins [40], Jones et al. [37], Gruber and Mullins [29], the development of grain boundary grooves, (Mullins [50, 51]; Srinivasan and Trivedi [69], Hackney and Ojard [31], Ratke and Vogel [60], Génin et al. [25], Vogel and Ratke [77]) and linear facet growth (Mullins [54]).

The linearized form (4) has failed to show some important effects of the nonlinearity. For a symmetric grain boundary groove evolving by surface diffusion only, the linear model predicts an infinite groove growth rate for a grain boundary groove with a zero dihedral angle. However, Broadbridge and Tritscher [14] show that a finite groove growth rate exists. Also, for a symmetric grain boundary groove evolving by evaporation-condensation only, the groove depth has a log growth rate as a function of the dihedral angle whereas the linearized equation has a linear growth rate (Karciga, [38]). Another failing, in relation to surface redistribution by surface diffusion only, is that the nonlinear equation amplifies the long wavelength "beating" of two closely-spaced short-wavelength perturbations whereas the linearized equation doesn't show this (Coleman et al. [18]).

We note that the linearized equation (4) is an integrable form of the governing equation (2) for a class of theoretical anisotropic materials which have constitutive relations evaporation coefficient,

$$
\begin{aligned}
\alpha(\theta) & =\alpha_{0}\left(1+y_{x}^{2}\right)^{-1 / 2} \\
& =\alpha_{0} \cos \theta,
\end{aligned}
$$

surface diffusivity,

$$
\begin{aligned}
D_{s}(\theta) & =D_{0}\left(1+y_{x}^{2}\right)^{-1 / 2} \\
& =D_{0} \sec \theta
\end{aligned}
$$


and surface tension,

$$
\gamma(\theta)=\gamma_{0}\left(C_{1} \cos \theta+C_{2} \sin \theta+\frac{1}{2} \sec \theta\right), \quad\left(C_{1}, C_{2} \text { arbitrary constants }\right)
$$

Equation (7) is the general solution of the Herring equation [33]

$$
\gamma(\theta)+\frac{d^{2} \gamma}{d \theta^{2}}=\gamma_{0}\left(1+y_{x}^{2}\right)^{3 / 2}=\gamma_{0} \sec ^{3} \theta
$$

In Appendix A, we show, using Herring's "tangent sphere" criterion [33], that this class of theoretical materials are stable with respect to the formation of an undulated structure or facets. This implies that their behavior is like a liquid crystal (Herring $[33,34])$. Polar graphs of these relations are presented in Figure 1. These show that isotropic materials are indeed modeled well if the slopes are relatively small, $\left|y_{x}\right|<$ $0.6(|\theta|<0.5 \mathrm{rad})$, which confirms what has been demonstrated by computation (Robertson [62]) and experiment (Mullins and Shewmon [53], Hilliard et al. [36], Gjostein [28]).

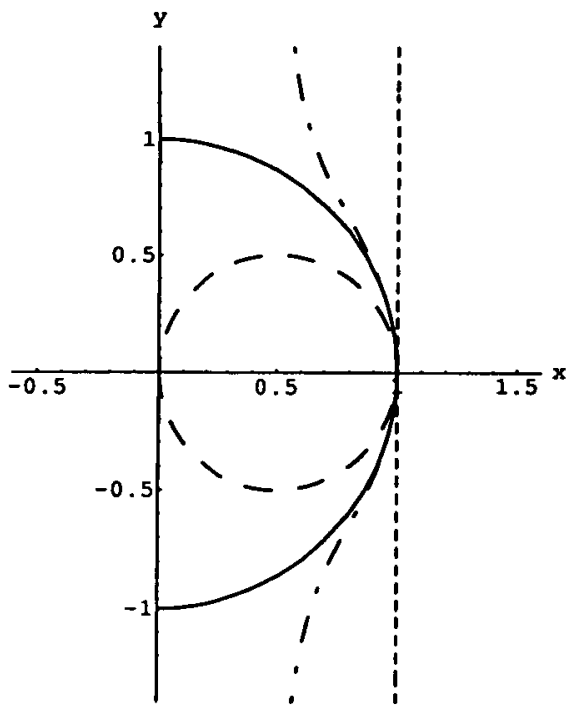

FIGURE 1. Polar graph of the constitutive relations (5)-(7) for the class of materials modeled by the linear equation (4),

- $-\ldots$ normalized evaporation coefficient, $\alpha / \alpha_{0}$;

$\ldots \ldots \ldots \ldots$ normalized surface diffusivity, $D / D_{0}$;

- - - - - normalized surface tension, $\gamma / \gamma_{0}$, with $C_{1}=1 / 2$ and $C_{2}=0$.

For comparison purposes, the circular section represents an isotropic material. 


\section{An integrable hierarchy of nonlinear evolution equations}

There exists another integrable form of governing equation (2) which models the nonlinearity of the equation for isotropic materials (3). It is suitable for test cases for numerical schemes and for a qualitative description of the evolution of the surface even when the surface slope is large.

Integrable partial differential equations which possess an infinite chain of LieBäcklund symmetries of arbitrarily high order are, in practice, transformable to linear equations, either directly by a change of variables, or indirectly by the inversescattering transform (Fokas [22], Bluman and Kumei [4], Mikhailov et al. [48], Broadbridge and Godfrey [12]). If $\Delta$ is a symmetry recursion operator for the class of evolution equation

$$
u_{t}=K\left(t, x, u, u_{1}, \ldots, u_{n}\right),
$$

where $u_{j}=\partial^{j} u(x, t) / \partial x^{j}$, then we may construct a hierarchy of evolution equations

$$
u_{t}=\Delta^{i} K, \quad i=0,1, \ldots
$$

each of which preserves the flow of (8) (Olver [58]).

We consider an hierarchy of higher-order evolution equations related to the wellknown integrable nonlinear diffusion equation $([72,23,44,45,3,63,64,35,66,67$, $9,68,10,13])$

$$
\Theta_{t}=\frac{\partial}{\partial x}\left[\Theta^{-2} \Theta_{x}\right]
$$

Equation (10) has Lie-Bäcklund symmetries (Bluman and Kumei [3]), in infinitesimal form

$$
t_{*}=t+\epsilon \quad \Theta_{*}=\Theta+\epsilon \Delta^{n} \frac{\partial}{\partial x}\left[\Theta^{-2} \Theta_{x}\right]+O\left(\epsilon^{2}\right),
$$

where $\Delta$ is the symmetry recursion operator

$$
\Delta=\frac{\partial^{2}}{\partial x^{2}} \circ \Theta^{-1} \int^{x}
$$

The hierarchy of evolution equations has the form

$$
\begin{aligned}
\Theta_{t} & =\Delta^{n} \frac{\partial}{\partial x}\left[\Theta^{-2} \Theta_{x}\right] \\
& =\left\{\begin{array}{l}
\frac{\partial}{\partial x}\left[\Theta^{-2} \Theta_{x}\right] \quad \text { for } n=0, \\
\frac{\partial^{2}}{\partial x^{2}} \circ\left[\Theta^{-1} \frac{\partial}{\partial x}\right] \circ \cdots \circ\left[\Theta^{-1} \frac{\partial}{\partial x}\right] \circ\left[\Theta^{-3} \Theta_{x}\right] \text { for } n=1,2, \ldots
\end{array}\right.
\end{aligned}
$$


Equation (10) transforms directly to the linear diffusion equation. One choice of linearizing transformation is a form of hodograph transformation (Clarkson et al. [17], Galaktionov et al. [24])

$$
\Theta=\left(u_{z}\right)^{-1}, \quad x=u(\tau, z), \quad t=\tau .
$$

Applying the chain rule when differentiating (14b) by $x$ and by $t$, we derive respectively

$$
\frac{\partial z}{\partial x}=\left(u_{z}\right)^{-1} \quad \text { and } \quad \frac{\partial z}{\partial t}=u_{\tau}\left(u_{z}\right)^{-1}
$$

The chain rule then yields

$$
\frac{\partial}{\partial x}=\left(u_{z}\right)^{-1} \frac{\partial}{\partial z}, \quad \frac{\partial}{\partial t}=\frac{\partial}{\partial \tau}-u_{\tau}\left(u_{z}\right)^{-1} \frac{\partial}{\partial z} .
$$

Substituting (14a) into (15) provides

$$
\frac{\partial \Theta}{\partial x}=-\left(u_{z}\right)^{-3} \frac{\partial^{2} u}{\partial z^{2}}=-\Theta^{2}\left(u_{z}\right)^{-1} \frac{\partial^{2} u}{\partial z^{2}}
$$

and

$$
\frac{\partial \Theta}{\partial t}=-\left(u_{z}\right)^{-2} \frac{\partial^{2} u}{\partial z \partial t}+u_{\tau}\left(u_{z}\right)^{-3} \frac{\partial^{2} u}{\partial z^{2}}=-u_{z}^{-1} \frac{\partial}{\partial z}\left(u_{\tau}\left(u_{z}\right)^{-1}\right) .
$$

Finally, substituting (15a), (16)-(17) into (10) gives the desired result

$$
\begin{aligned}
-u_{z}^{-1} \frac{\partial}{\partial z}\left(u_{\tau}\left(u_{z}\right)^{-1}\right) & =-u_{z}^{-1} \frac{\partial}{\partial z}\left(u_{z}^{-1} u_{z z}\right) \\
\Longrightarrow \quad u_{\tau} & =u_{z z} .
\end{aligned}
$$

Subsequently, the infinity of flows (13) which preserve the flow of (10) ensure that the higher-order nonlinear evolution equations (13) transform to linear equations.

Not only are each of the members of the hierarchy (13) linearizable, we also have the surprising result that any linear combination of the members of the hierarchy are linearizable!

PROPOSITION 1 (Rogers [65]). The nonlinear equation

$$
\Theta_{t}=q_{0}(t) \frac{\partial}{\partial x}\left[\Theta^{-2} \Theta_{x}\right]+\sum_{i=1}^{n} q_{i}(t) \frac{\partial^{2}}{\partial x^{2}} \circ\left[\Theta^{-1} \frac{\partial}{\partial x}\right]^{i-1} \circ\left[\Theta^{-3} \Theta_{x}\right],
$$

where $q_{i}$ are arbitrary functions of $t$, is linearized by the transformation (14). 
ProOF. Substituting (15a), (16)-(17) into (18) and noting that $\Theta=\left(u_{2}\right)^{-1}$ implies

$$
-u_{z}^{-1} \frac{\partial}{\partial z}\left(u_{\tau}\left(u_{z}\right)^{-1}\right)=-q_{0}(\tau) u_{z}^{-1} \frac{\partial}{\partial z}\left(u_{z}^{-1} u_{z z}\right)-\sum_{i=1}^{n} q_{i}(\tau) u_{z}^{-1} \frac{\partial}{\partial z} \circ u_{z}^{-1} \frac{\partial^{i-1}}{\partial z^{i-1}} u_{z z}
$$

and the linear equation

$$
u_{\mathrm{r}}=\sum_{i=0}^{n} q_{i}(\tau) \frac{\partial^{i+2}}{\partial z^{i+2}} u
$$

follows.

Various members of (18) have been applied to surface redistribution previously. A form of the $n=0$ member was used to model the evolution of an initialy ramped surface by evaporation-condensation (Broadbridge [11]). The $n=2$ member was used for the qualitative and quantitative description of the development of a symmetric grain boundary groove by surface diffusion for an isotropic material (Broadbridge and Tritscher [14], Tritscher and Broadbridge [76]).

However, if we choose the members $i=0,2$ with $q_{0}(t)=A \bar{\alpha}_{0} \bar{\gamma}_{0}$ and $q_{2}(t)=$ $-B \bar{D}_{0} \bar{\gamma}_{0}$, where $\bar{\alpha}_{0}, \bar{D}_{0}$ and $\bar{\gamma}_{0}$ are weights for the evaporation coefficient, surface diffusivity, and surface tension respectively, an integrable governing equation which has a form similar to (3) is created whereby we incorporate concomitant evaporationcondensation and surface diffusion. In terms of the potential $y=a \int^{x} \Theta d x-$ $b x,(a, b$ constants), this equation is

$$
y_{t}=A \frac{\bar{\alpha}_{0} \bar{\gamma}_{0} a^{2} y_{x x}}{\left(b+y_{x}\right)^{2}}-B \frac{\partial}{\partial x}\left\{\frac{\bar{D}_{0} a}{b+y_{x}} \frac{\partial}{\partial x}\left[\frac{\bar{\gamma}_{0} a^{3} y_{x x}}{\left(b+y_{x}\right)^{3}}\right]\right\} .
$$

We have as constitutive relations:

evaporation coefficient,

$$
\alpha(\theta)=\alpha_{0} \frac{\bar{\alpha}_{0}\left(b+y_{x}\right)}{a\left(1+y_{x}^{2}\right)^{1 / 2}}=\alpha_{0} \frac{\bar{\alpha}_{0}\left(1+b^{2}\right)^{1 / 2}}{a} \cos (\theta-\operatorname{arccot} b),
$$

surface diffusivity,

$$
D_{s}(\theta)=D_{0} \frac{\bar{D}_{0} a\left(1+y_{x}^{2}\right)^{1 / 2}}{\left(b+y_{x}\right)}=D_{0} \frac{\bar{D}_{0} a}{\left(1+b^{2}\right)^{1 / 2}} \sec (\theta-\operatorname{arccot} b),
$$

and surface tension,

$$
\begin{aligned}
\gamma(\theta)=\gamma_{0} \frac{\bar{\gamma}_{0} a^{3}}{\left(1+b^{2}\right)^{3 / 2}}( & C_{1} \cos (\theta-\operatorname{arccot} b) \\
& \left.+C_{2} \sin (\theta-\operatorname{arccot} b)+\frac{1}{2} \sec (\theta-\operatorname{arccot} b)\right),
\end{aligned}
$$


where $C_{1}, C_{2}$ are arbitrary constants. Equation (22) is the general solution of

$$
\gamma(\theta)+\frac{d^{2} \gamma}{d \theta^{2}}=\gamma_{0} \frac{\bar{\gamma}_{0} a^{3}\left(1+y_{x}^{2}\right)^{3 / 2}}{\left(b+y_{x}\right)^{3}}=\gamma_{0} \frac{\bar{\gamma}_{0} a^{3}}{\left(1+b^{2}\right)^{3 / 2}} \sec ^{3}(\theta-\operatorname{arccot} b) .
$$

So, surprisingly, the nonlinear equation (19) models the same class of theoretical materials as the linear equation (4)! However, we now have the freedom to arbitrarily orient the anisotropic material.

The linear equation satisfactorily modeled isotropic materials provided the surface inclination varied by less than approximately $\pi / 4 \mathrm{rad}$ about the line $y=0$. Since the nonlinear equation represents the same class of materials as the linear equation but with an arbitrary orientation, then we may model isotropic materials where the inclination varies by approximately $\pi / 4$ rad about an arbitrary line. Here we apply the nonlinear equation (19) to the evolution of an initially-ramped surface by the concomitant action of evaporation-condensation and surface diffusion. Also, a comparison with a numerical solution for an isotropic material is made.

\section{Evolution of a ramped surface}

We consider the evolution of an extended surface that is ramped with an arbitrary inclination $\Psi$ and rise of $2 h$. By an appropriate choice of axes, the initial value problem may be formulated with the initial condition

$$
y(x, 0)=\left\{\begin{array}{lll}
m x & \text { for } & 0 \leq x<h / m, \\
h & \text { for } & x \geq h / m,
\end{array}\right.
$$

where $m=\tan \Psi$. By symmetry we only consider the region $x \geq 0$. The antisymmetric initial condition implies that, at $x=0$, the curvature and velocity vanish so that

$$
\frac{\partial^{2 n} y}{\partial x^{2 n}}=0 \quad \text { at } \quad x=0 \quad \text { for } \quad n=0,1
$$

At early time surface diffusion is dominant [32], so a convenient scaling [19] is

$$
y^{*}=h^{-1} y, \quad x^{*}=h^{-1} x, \quad \text { and } \quad t^{*}=h^{-4} \bar{D}_{0} \bar{\gamma}_{0} B t .
$$

Equation (19) transforms to

$$
y_{t^{*}}^{*}=v^{*} \frac{a^{2} y_{x^{*} x^{*}}^{*}}{\left(b+y_{x^{*}}^{*}\right)^{2}}-\frac{\partial}{\partial x^{*}}\left\{\frac{a}{b+y_{x^{*}}^{*}} \frac{\partial}{\partial x^{*}}\left[\frac{a^{3} y_{x^{*} x^{*}}^{*}}{\left(b+y_{x^{*}}^{*}\right)^{3}}\right]\right\},
$$


where $v^{*}=v \bar{\alpha}_{0} \bar{D}_{0}^{-1}$ with $\nu=h^{2} A / B$ being the dimensionless ratio of the object length to the diffusive length. We have initial condition

$$
y^{*}\left(x^{*}, 0\right)=\left\{\begin{array}{lll}
m x^{*} & \text { for } & 0 \leq x^{*}<1 / m \\
1 & \text { for } & x^{*} \geq 1 / m
\end{array}\right.
$$

and at the origin

$$
\frac{\partial^{2 n} y^{*}}{\partial x^{* 2 n}}=0 \quad \text { at } \quad x^{*}=0 \quad \text { for } \quad n=0,1 .
$$

Though the hodograph transformation (14) gives a means of transforming (26) to linear form, a linearization procedure derived by Knight [44], who modified a transformation of Storm [72], is more convenient for the boundary conditions. The problem is linearized by

$$
\begin{aligned}
& \eta=a\left(b+y_{x^{*}}^{*}\right)^{-1}-a / b, \\
& \chi=\int_{0}^{x^{*}}(\eta+a / b)^{-1} d x^{*}, \\
& \tau=t^{*}
\end{aligned}
$$

In Appendix B, we derive the canonical form

$$
\frac{\partial^{4} \eta}{\partial \chi^{4}}-\nu^{*} \frac{\partial^{2} \eta}{\partial \chi^{2}}+\frac{\partial \eta}{\partial \tau}=0
$$

The initial condition remains linear. At $t^{*}=0$

$$
\begin{aligned}
\chi & =a^{-1}\left(y^{*}\left(x^{*}, 0\right)+b x^{*}\right) \\
& =\left\{\begin{array}{lll}
a^{-1}(m+b) x^{*} & \text { for } & 0 \leq x^{*}<1 / m, \\
a^{-1}(1+b) x^{*} & \text { for } & x^{*} \geq 1 / m .
\end{array}\right.
\end{aligned}
$$

Inverting (33) and noting that $\partial x^{*} / \partial \chi=\eta+a / b$, the initial condition transforms to

$$
\eta(\chi, 0)=\left\{\begin{array}{lll}
-a m b^{-1}(b+m)^{-1} & \text { for } & 0 \leq \chi<(b+m) /(a m), \\
0 & \text { for } \quad \chi \geq(b+m) /(a m)
\end{array}\right.
$$

At $x=0$

$$
\frac{\partial^{n} \eta}{\partial \chi^{n}}=0, \quad \text { for } \quad n=1,3
$$


Employing the Fourier cosine transformation, we derive the solution

$$
\eta(\chi, \tau)=-\frac{2 a m}{\pi b(b+m)} \int_{0}^{\infty} s^{-1} e^{-\left(s^{4}+v^{*} s^{2}\right) \tau} \sin \left(\frac{(b+m)}{a m} s\right) \cos (\chi s) d s
$$

for $\eta$. Inverting (29)-(31) and a change of parameterization yields a solution $\left(t^{*}\left(\tau^{*}\right), x^{*}\left(\phi, \tau^{*}\right), y^{*}\left(\phi, \tau^{*}\right)\right)$ :

$$
\begin{aligned}
y^{*} & =\int_{0}^{x^{*}} a(\eta+a / b)^{-1}-b d x^{*} \\
& =a \chi-b \int_{0}^{x} \eta+a / b d \chi \\
& =\frac{2 a m}{\pi(b+m)} \int_{0}^{\infty} s^{-2} e^{-\left(s^{4}+v^{*} s^{2}\right) \tau} \sin \left(\frac{(b+m)}{a m} s\right) \sin (\chi s) d s \\
& =\frac{2 \tau^{* 1 / 4}}{\pi} \int_{0}^{\infty} s^{-2} e^{-s^{4}-\tau^{* 1 / 2} \frac{\bar{a}_{0}}{D_{0}}\left(\frac{b+m}{a m}\right)^{2} s^{2}} \sin \left(\frac{s}{\tau^{* 1 / 4}}\right) \sin \left(\frac{\phi}{\tau^{* 1 / 4}} s\right) d s, \\
x^{*} & =-\frac{y^{*}}{b}+\frac{b+m}{b m} \phi
\end{aligned}
$$

and

$$
t^{*}=\left(\frac{b+m}{a m}\right)^{4} \tau^{*}
$$

By a Cauchy product of the appropriate two series, we may express (37) in series form as

$$
\begin{aligned}
y^{*}= & \frac{\phi}{\pi \tau^{* 1 / 4}} \sum_{n=0}^{\infty}(-1)^{n} \Gamma(n / 2+1 / 4) \\
& \times \sum_{i=0}^{n} \frac{\tau^{* n / 2-i}\left[\nu^{*}\left(\frac{b+m}{a m}\right)^{2}\right]^{n-i}}{(2 i+2) !(n-i) !} \sum_{j=0}^{i}(1-\phi)^{2 i-2 j}(1+\phi)^{2 j} .
\end{aligned}
$$

This series is absolutely convergent for $\phi \in \Re$ and $\tau^{*} \in \mathfrak{M}-\{0\}$ [75].

We note that the case where $a, b \rightarrow \infty$, with the weights $\bar{\alpha}_{0}, \bar{D}_{0}$, and $\bar{\gamma}_{0}$ adjusted accordingly, yields the solution when the small-slope approximation is assumed (Mullins [55]). For comparison and entirety, we present the solution when evaporation-condensation only is operative, $B=0$, (Broadbridge [11])

$$
\begin{aligned}
y^{*}= & \frac{1}{2}(\phi+1) \operatorname{erf}\left(\frac{1}{2 \tau_{A}^{* 1 / 2}}[\phi+1]\right)-\frac{1}{2}(\phi-1) \operatorname{erf}\left(\frac{1}{2 \tau_{A}^{* 1 / 2}}[\phi-1]\right) \\
& +\frac{\tau_{A}^{* 1 / 2}}{\pi^{1 / 2}}\left\{\exp \left(-\frac{1}{4 \tau_{A}^{*}}[\phi+1]^{2}\right)-\exp \left(-\frac{1}{4 \tau_{A}^{*}}[\phi-1]^{2}\right)\right\}, \\
x^{*}= & -\frac{y^{*}}{b}+\frac{b+m}{b m} \phi
\end{aligned}
$$


and

$$
h^{-2} \bar{\alpha}_{0} \bar{\gamma}_{0} A t=\left(\frac{b+m}{a m}\right)^{2} \tau_{A}^{*}
$$

Graphical presentation of the solution for the cases evaporation-condensation only, surface diffusion only and concomitant evaporation-condensation and surface diffusion with $\nu \bar{\alpha}_{0} \bar{D}_{0}^{-1}(b+m)^{2}(a m)^{-2}=1$ are shown in Figure 2. We also display, in Figure 3, the graph of scaled slope at the origin versus scaled time. For conciseness, the surface profiles and scaled slope are shown in the parameter space $\left(\tau^{*}, \phi, y^{*}\right)$. However, the graphs show actual profiles and slopes if we assume the small slope approximation, $a, b \rightarrow \infty$.

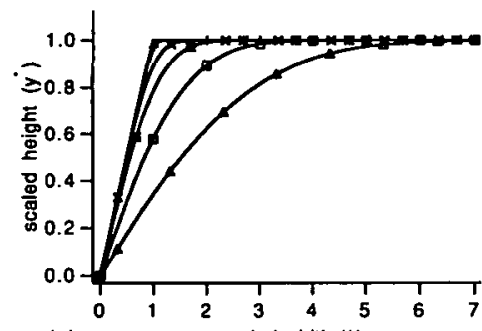

(a)

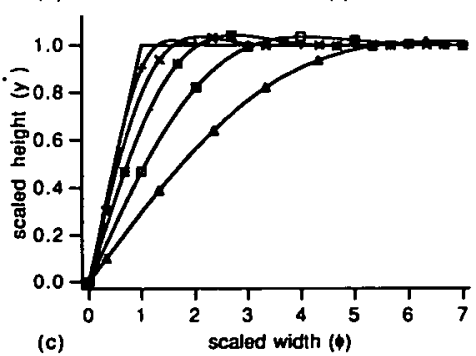

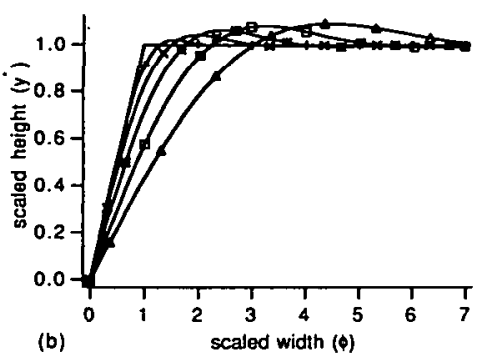

(b)

scaled width (\$)

FIGURE 2. Graph of scaled height $y^{*}$ versus scaled width $\phi=b m(b+m)^{-1}\left(x^{*}+y^{*} b^{-1}\right)$ for (a) evaporation-condensation only, (b) surface diffusion only and (c) concomitant evaporation-condensation and surface diffusion with

$\nu \bar{\alpha}_{0} \bar{D}_{0}^{-1}(b+m)^{2}(a m)^{-2}=1$.

$-\tau^{\dagger}=0 ;-十 \tau^{\dagger}=0.00311 ; \rightarrow \quad \times \quad \tau^{\dagger}=0.0372$;

$\rightarrow \ldots-\tau^{\dagger}=0.149$

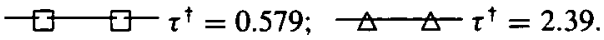

$\tau^{\dagger}$ is scaled time, $\tau^{\dagger}=(a m)^{2}(b+m)^{-2} \bar{\alpha}_{0} \bar{\gamma}_{0} h^{-2} A t$ for evaporation-condensation, or $\tau^{\dagger}=(a m)^{4}(b+$ $m)^{-4} \bar{D}_{0} \bar{\gamma}_{0} h^{-4} B t$ for surface diffusion or concomitant evaporation-condensation and surface diffusion.

There are some distinctive differences in the surface profiles among the modes of surface redistribution. These are that the surface height has no local maxima when evaporation-condensation only is operative, whereas for surface diffusion the surface height exhibits local maxima for which the predominant one approaches a dimensionless height, $y^{*}$, of 1.104 in the limit as time approaches infinity. For concomitant evaporation-condensation and surface diffusion the surface height reaches a 


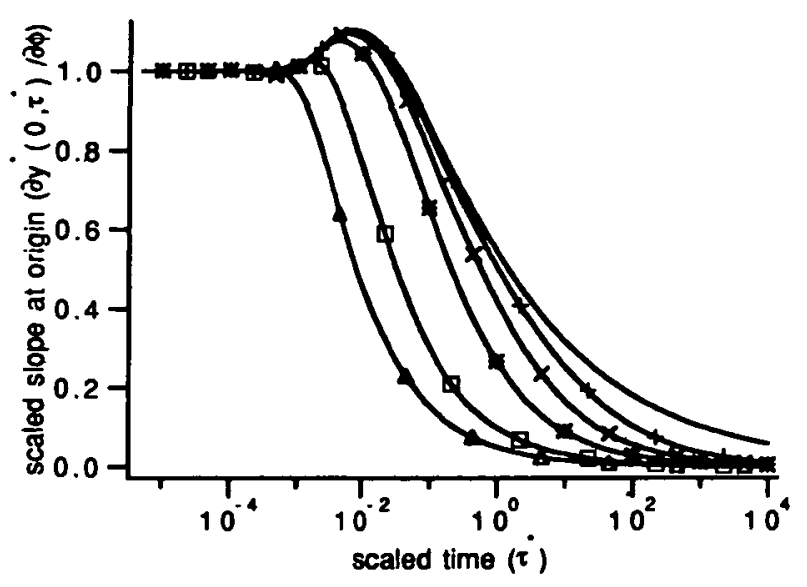

FIGURE 3. Graph of scaled slope at origin $y_{\phi}^{*}\left(0, \tau^{*}\right)=m^{-1}(b+m) y_{x^{*}}^{*}\left(0, \tau^{*}\right)\left(b+y_{x^{*}}^{*}\left(0, \tau^{*}\right)\right)^{-1}$ versus scaled time $\tau^{*}=(a m)^{4}(b+m)^{-4} \bar{D}_{0} \bar{\gamma}_{0} h^{-4} B t$.

$\longrightarrow v^{\dagger}=0$ (surface diffusion only); $\longrightarrow \perp v^{\dagger}=0.25 ; \longrightarrow \times \quad v^{\dagger}=1$; - * $-v^{\dagger}=4 ; \square \quad \square v^{\dagger}=32 ; \triangle \Delta v^{\dagger}=128$.

$\nu^{\dagger}$ is a compound parameter, $\nu^{\dagger}=\nu \bar{\alpha}_{0} \bar{D}_{0}^{-1}(a m)^{-2}(b+m)^{2}$.

global maximum, which is less than 1.104, with the limiting profile approaching the profile of evaporation-condensation only. We show the derivation of these limits in Appendix C.

Implicit in the graph of the scaled slope at the origin, Figure 3, is that, if surface diffusion is present, the profile for some initial inclinations will develop a vertical tangent at the origin. The scaled slope $y_{\phi}^{*}\left(0, \tau^{*}\right)$ shows a maximum of 1.104 . From (37)-(39),

$$
y_{x^{*}}^{*}\left(0, t^{*}\right)=b y_{\phi}^{*}\left(0, \tau^{*}\right)\left(b / m+1-y_{\phi}^{*}\left(0, \tau^{*}\right)\right)^{-1} .
$$

This implies that a vertical tangent may develop if $m \gtrsim b / 0.104$. If the initial inclination is large and we wish to approximate an isotropic material, then $b \simeq 1$ (see Section 5), hence a vertical tangent may develop if $m \gtrsim 10$ which corresponds to an initial inclination of $\Psi \gtrsim 84^{\circ}$. If $b \rightarrow \infty$, which is the solution when the small slope approximation is assumed, then no vertical tangent develops.

\section{Comparison with a numerical solution for an isotropic material}

A numerical solution for the evolution of a ramp for an isotropic material is given in Lee [47]. The method of lines with a stiff ordinary differential equation solver was 
employed and the fidelity of the method was verified by applying the scheme to the theoretical anisotropic material.

We make our comparison using a ramp initially inclined at $\pi / 4 \mathrm{rad}$. This is chosen, not because we felt that this was the limit for which our theoretical anisotropic material would give a reasonable approximation for an isotropic material, but rather, it was imposed by instability in the numerical scheme. In the calculation for the theoretical anisotropic material, we choose the following values for the scales and parameters in the constitutive relations (20)-(22): $\bar{\alpha}_{0}=a /\left(1+b^{2}\right)^{-1 / 2} ; \bar{D}_{0}=1 ; \bar{\gamma}_{0}=\left(1+b^{2}\right) / a^{2}$; $C_{1}=\left(1+b^{2}\right) /\left(2 a^{2}\right) ; C_{2}=0$ and $a=b=\cot (\pi / 8)$. The relations are explicitly presented below:

evaporation coefficient $(\leq 1)$,

$$
\alpha(\theta)=\alpha_{0} \cos (\theta-\pi / 8)
$$

surface diffusivity,

$$
D_{s}(\theta)=D_{0} \cos (\pi / 8) \sec (\theta-\pi / 8),
$$

and surface tension,

$$
\gamma(\theta)=\frac{\gamma_{0}}{2}\{\sec (\pi / 8) \cos (\theta-\pi / 8)+\cos (\pi / 8) \sec (\theta-\pi / 8)\},
$$

where we note that the surface tension satisfies the requirement of a minimum at $\theta=0$.

This orients our theoretical material so that the line of symmetry is inclined at $\theta=\pi / 8$. Over the range of inclinations spanned, these constitutive relations give a relatively close representation for an isotropic material. A graphical presentation of these relations, compared to the constitutive relations for an isotropic material, is shown in Figure 4. Near the extremes of inclination, the surface diffusivity and surface tension agree. The restriction that the evaporation coefficient be less than or equal to one, necessitates a small compromise however.

Graphs of the surface profiles, and the inclination at the origin versus dimensionless time, for an isotropic material and our theoretical anisotropic material are shown in Figures 5 and 6 respectively. Displayed are the profiles for evaporation-condensation only, surface diffusion only and concomitant evaporation-condensation and surface diffusion with $v=1$. The inclination at the origin is calculated for a range of values of $v$ from 0 (surface diffusion only) to 16 (evaporation-condensation dominant). We comment that the profiles are remarkably close! The maximum absolute difference in any of the profiles is a mere 0.015 dimensionless units. 


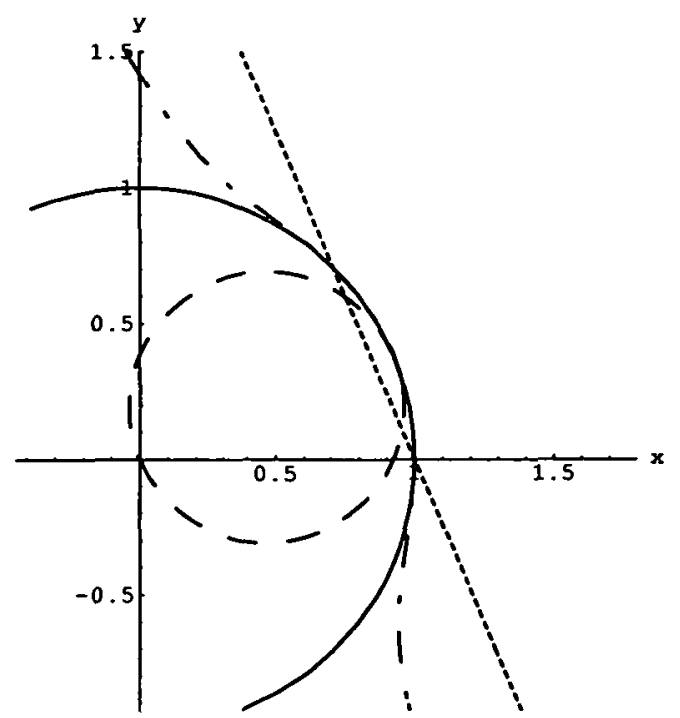

FIGURE 4. Polar graph of the constitutive relations (44)-(46).

- $\ldots$ - normalized evaporation coefficient, $\alpha / \alpha_{0}$;

$\cdots \cdots \cdots \cdots$ normalized surface diffusivity, $D / D_{0}$;

- . - - - - normalized surface tension, $\gamma / \gamma_{0}$.

For comparison purposes, the circular section represents an isotropic material.

\section{Conclusions}

We have derived a new integrable nonlinear equation which models the surface evolution of an arbitrarily-oriented theoretical anisotropic material by the concomitant action of evaporation-condensation and surface diffusion. This theoretical anisotropic material behaves like a liquid crystal and shares the same constitutive relations as those of the constant coefficient linear equation used in the small slope approximation for isotropic materials. The integrable nonlinear equation may be used to advantage as test cases for numerical schemes. Its form has many attributes of the nonlinear governing equation for an isotropic material.

Closed-form solutions are constructed, where the theoretical anisotropic material may be arbitrarily oriented, for the evolution of a ramped surface by concomitant evaporation-condensation and surface diffusion. The solution for the ramped surface showed, unlike the solution when the small slope approximation is assumed, that a vertical tangent may develop for the case where the initial inclination is large $\left(\gtrsim 84^{\circ}\right)$. We have given a solution for a specific initial condition. However, surface redistribution for a general antisymmetric initial condition, such that the initial surface inclination deviates by no more than $\pi / 4 \mathrm{rad}$ about the line of symmetry of the theoretical anisotropic material, may be accommodated. 

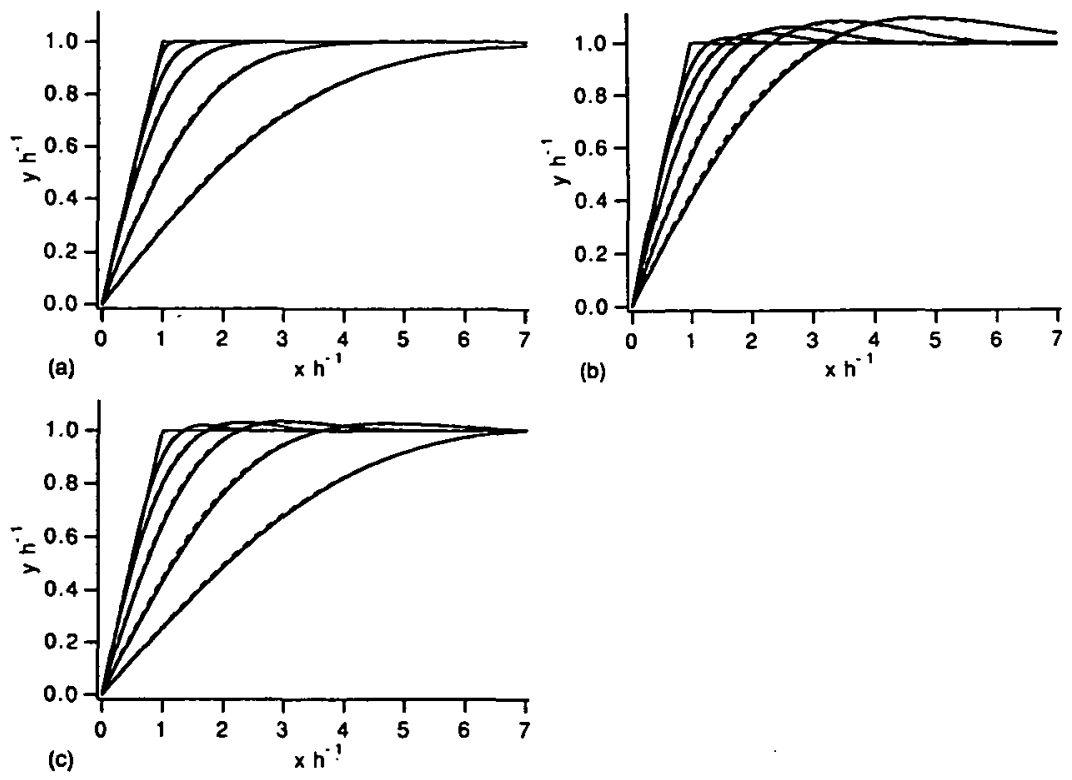

FIGURE 5. Surface profiles for (a) evaporation-condensation only, (b) surface diffusion only and (c) concomitant evaporation-condensation and surface diffusion with $v=1$. The ramp is initialy inclined at $\pi / 4 \mathrm{rad}$.

isotropic material; _ - - - theoretical anisotropic material; Dimensionless times from left to right $t^{\dagger}=0,0.005,0.061,0.242,0.970,3.88 . t^{\dagger}=h^{-2} A t$ for evaporationcondensation, or $t^{\dagger}=h^{-4} \mathrm{Bt}$ for surface diffusion or concomitant evaporation-condensation and surface diffusion.

It was stated earlier that the transport modes evaporation-condensation and surface diffusion yield opposing groove growth rates in the development of grain boundary grooves. Karciga [38] has shown that the transport mode evaporation-condensation only has an unbounded groove growth rate for a groove with a zero dihedral angle, but the the groove growth rate is finite for a groove developing by surface diffusion only. Hence, the question of whether a finite groove growth rate exists by the concomitant action of evaporation-condensation and surface diffusion. Physically, a finite growth rate is suspected to be the case, as evaporation may be ignored near the root of the groove where the opposite walls are only a few atomic diameters apart. However, our new governing equation appears to be unable to assist in the resolution of this problem because of a nonlinearity of the flux gradient at the origin. In principle, a solution may be constructed if we specify the form of the flux gradient at the origin, however, this forces the form of the flux at the origin, which doesn't suit our purposes. 


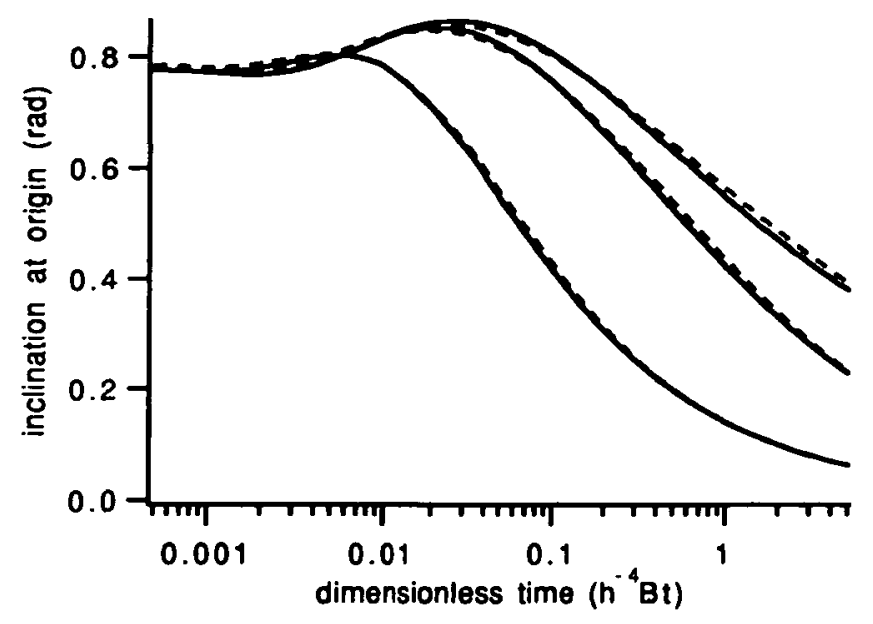

FIGURE 6. Graph of inclination at the origin versus dimensionless time for parameter $v=0,1,16$ from top down. The ramp is initialy inclined at $\pi / 4 \mathrm{rad}$.

isotropic material; - - - - theoretical anisotropic material.

\section{Acknowledgements}

The author thanks Professor P. Broadbridge for many helpful discussions and Professor A. J. Roberts for the suggestion to compare the analytic solution to numerical results. Also, thanks to Mathew Lee for lending his computer software for the numerical work. The author acknowledges the generous financial support provided by the University of Wollongong and the Illawarra Technology Corporation.

\section{Appendix A}

We show that the class of theoretical anisotropic materials modeled by the linearized equation (4) are stable with respect to the formation of an undulated structure or facets. The stability is readily confirmed using the "tangent sphere" criterion by Herring [33], which states that the surface with orientation in the neighborhood of the point $\left(P, \theta_{P}\right)$ on the $\gamma$-plot is stable if, and only if, the $\gamma$-plot nowhere passes inside the sphere drawn tangent to $\left(P, \theta_{P}\right)$ and through the origin. For our purposes, only a planar section through the $\gamma$-plot, called a $\gamma_{2}$-plot [55], need be considered, so Herring's condition simplifies to the construction of a tangent circle at $\left(P, \theta_{P}\right)$ of the $\gamma_{2}$-plot.

We follow the notation given by Gjostein [27]. The equation of a circle, given in 
polar coordinates $(R, \theta)$, which passes through the origin is

$$
R=2(\bar{h} \sin \theta+\bar{k} \cos \theta),
$$

where $\bar{h}, \bar{k}$ are the $x$ and $y$ coordinates of the center of the circle. The requirement that the circle is tangent to the $\gamma_{2}$-plot at the point $\left(P, \theta_{P}\right)$ leads to

$$
\begin{aligned}
\gamma\left(\theta_{P}\right)=R\left(\theta_{P}\right) & =2\left(\bar{h} \sin \theta_{P}+\bar{k} \cos \theta_{P}\right) \\
\text { and } \quad \frac{d \gamma}{d \theta}\left(\theta_{P}\right)=\frac{d R}{d \theta}\left(\theta_{P}\right) & =2\left(\bar{h} \cos \theta_{P}-\bar{k} \sin \theta_{P}\right) .
\end{aligned}
$$

Solving for $\bar{h}, \bar{k}$ yields

$$
\begin{aligned}
& \bar{h}=\frac{1}{2}\left(\gamma\left(\theta_{P}\right) \sin \theta_{P}+\frac{d \gamma}{d \theta}\left(\theta_{P}\right) \cos \theta_{P}\right), \\
& \bar{k}=\frac{1}{2}\left(\gamma\left(\theta_{P}\right) \cos \theta_{P}-\frac{d \gamma}{d \theta}\left(\theta_{P}\right) \sin \theta_{P}\right) .
\end{aligned}
$$

From (7), the surface tension and torque at $\left(P, \theta_{P}\right)$ is given by

$$
\text { and } \begin{aligned}
\gamma\left(\theta_{P}\right) & =\gamma_{0}\left(C_{1} \cos \theta_{P}+C_{2} \sin \theta_{P}+\frac{1}{2} \sec \theta_{P}\right) \\
\frac{d \gamma}{d \theta}\left(\theta_{P}\right) & =\gamma_{0}\left(-C_{1} \sin \theta_{P}+C_{2} \cos \theta_{P}+\frac{1}{2} \sec \theta_{P} \tan \theta_{P}\right) \text {. }
\end{aligned}
$$

Substituting (A2)-(A5) into (A1) yields the equation

$$
R(\theta)=\gamma_{0}\left(C_{1} \cos \theta+C_{2} \sin \theta+\tan \theta_{P} \sin \theta+\frac{1}{2} \cos \theta-\frac{1}{2} \tan ^{2} \theta_{P} \cos \theta\right)
$$

of the tangent circle. We show that $R(\theta) \leq \gamma(\theta)$. By symmetry we need only consider $\theta \in(-\pi / 2, \pi / 2)$. Comparing (7) and (A6), it is sufficient to show that

$$
2 \tan \theta_{P} \sin \theta \cos \theta+\left(1-\tan ^{2} \theta_{P}\right) \cos ^{2} \theta \leq 1 .
$$

Equation (A7) implies

$$
\begin{aligned}
\tan \theta_{P} & \sin (2 \theta)+\frac{1}{2}\left(1-\tan ^{2} \theta_{P}\right)(\cos (2 \theta)+1) \\
& =\tan \theta_{P} \sin (2 \theta)+\frac{1}{2}\left(1-\tan ^{2} \theta_{P}\right) \cos (2 \theta)+\frac{1}{2}-\frac{1}{2} \tan ^{2} \theta_{P} \\
& =\left[\tan ^{2} \theta_{P}+\frac{1}{4}\left(1-\tan ^{2} \theta_{P}\right)^{2}\right]^{1 / 2} \cos \left(2 \theta-2 \theta_{P}\right)+\frac{1}{2}-\frac{1}{2} \tan ^{2} \theta_{P} \\
& =\frac{1}{2}\left(1+\tan ^{2} \theta_{P}\right) \cos \left(2 \theta-2 \theta_{P}\right)+\frac{1}{2}-\frac{1}{2} \tan ^{2} \theta_{P} \\
& \leq 1,
\end{aligned}
$$

because $\cos \left(2 \theta-2 \theta_{P}\right) \leq 1$. This completes the proof. 


\section{Appendix B}

We detail the transformation of the nonlinear equation (26) to the linear canonical form (32). Equation (26) may be expressed as

$$
\Theta_{t^{*}}=-\frac{\partial^{2} J}{\partial x^{2}}
$$

where

$$
\Theta=\frac{\partial y^{*}}{\partial x^{*}}
$$

and

$$
\begin{aligned}
J & =v^{*} \frac{a^{2}}{b+\Theta_{x^{*}}}+\frac{a}{b+\Theta_{x^{*}}} \frac{\partial}{\partial x^{*}}\left[\frac{a^{3} \Theta_{x^{*}}}{\left(b+\Theta_{x^{*}}\right)^{3}}\right], \\
& =v^{*} a(\eta+a / b)-a(\eta+a / b) \frac{\partial}{\partial x^{*}}\left[(\eta+a / b) \eta_{x^{*}}\right]
\end{aligned}
$$

By (29). From (30),

$$
\frac{\partial}{\partial x^{*}}=(\eta+a / b)^{-1} \frac{\partial}{\partial \chi}
$$

so (B3) transforms to

$$
J=v^{*} a(\eta+a / b)-a \eta_{x x}
$$

Hence

$$
\frac{\partial J}{\partial x^{*}}=-a(\eta+a / b)^{-1}\left(\eta_{x x \chi}-v^{*} \eta_{X}\right)
$$

and finally

$$
\begin{aligned}
\frac{\partial^{2} J}{\partial x^{* 2}}=-a(\eta+a / b)^{-2}\left[\eta_{x x x x}\right. & -(\eta+a / b)^{-1} \eta_{x} \eta_{x x x} \\
& \left.-\eta_{x x}+\nu^{*}(\eta+a / b)^{-1}\left(\eta_{x}\right)^{2}\right] .
\end{aligned}
$$

Next, we transform the left-hand side of (B1). By (29),

$$
\Theta_{r^{*}}=-a(\eta+a / b)^{-2} \eta_{i^{*}}
$$


and

$$
\begin{array}{rlr}
\eta_{i^{*}} & =\eta_{\tau}+a^{-1} \eta_{x} \int_{0}^{x^{*}} \frac{\partial \Theta}{\partial t} d x \quad \text { from (29)-(30) } \\
& =\eta_{\tau}+a^{-1} \eta_{x} \int_{0}^{x^{*}}-\frac{\partial^{2} J}{\partial x^{* 2}} d x \quad \text { by (B1) } \\
& =\eta_{\tau}-a^{-1} \eta_{x}\left[\frac{\partial J}{\partial x^{*}}\right]_{0}^{x^{*}} \\
& =\eta_{\tau}-(\eta+a / b)^{-1} \eta_{x}\left(\eta_{x x x}-v^{*} \eta_{x}\right) \quad \text { by (B6), }
\end{array}
$$

where we note that, from the initial condition, the velocity at the origin vanishes

$$
\left.\frac{\partial J}{\partial x^{*}}\right|_{x^{*}=0}=-\left.\frac{\partial y^{*}}{\partial t^{*}}\right|_{x^{*}=0}=0
$$

Substituting (B7), and the resultant of (B9), (B8), into (B1) yields the linear canonical form (32).

\section{Appendix C}

When surface diffusion only is operative, we show that, as time approaches infinity, the dimensionless rise approaches 2.209 , whereas, if evaporation-condensation is present, the dimensionless rise approaches 2 . We first consider the case of surface diffusion only, $A=0$.

$$
\begin{aligned}
\lim _{v^{*} \rightarrow \infty} y^{*} & =\lim _{\tau^{*} \rightarrow \infty} \frac{2 \tau^{* 1 / 4}}{\pi} \int_{0}^{\infty} s^{-2} e^{-s^{4}} \sin \left(\frac{s}{\tau^{* 1 / 4}}\right) \sin \left(\frac{\phi}{\tau^{* 1 / 4}} s\right) d s, \quad \text { from (37) } \\
& =\frac{2}{\pi} \int_{0}^{\infty} s^{-1} e^{-s^{4}} \lim _{\tau^{*} \rightarrow \infty} \frac{\tau^{* 1 / 4}}{s} \sin \left(\frac{s}{\tau^{* 1 / 4}}\right) \sin (\Phi s) d s \\
& =\frac{2}{\pi} \int_{0}^{\infty} s^{-1} e^{-s^{4}} \sin (\Phi s) d s
\end{aligned}
$$

where $\Phi=\phi \tau^{*-1 / 4}$ is finite. The function (C1) has the form of the self similar solution for the development of the ramp modeled by the linear equation (4) when $m \rightarrow \infty$ (Mullins [55]). The graph exhibits a global maximum of 1.1044 to yield a dimensionless rise of 2.209 . 
Now consider when evaporation-condensation is present.

$$
\begin{aligned}
\lim _{t^{*} \rightarrow \infty} y^{*} & =\lim _{\tau^{*} \rightarrow \infty} \frac{2 \tau^{* 1 / 4}}{\pi} \int_{0}^{\infty} s^{-2} e^{-s^{4}-v^{\dagger} \tau^{* 1 / 2} s^{2}} \sin \left(\frac{s}{\tau^{* 1 / 4}}\right) \sin \left(\frac{\phi}{\tau^{* 1 / 4}} s\right) d s \text { from (37) } \\
& =\lim _{\tau^{*} \rightarrow \infty} \frac{2}{\pi} \int_{0}^{\infty} u^{-2} e^{-\tau^{*-1} \Phi^{* 4} u^{4}-v^{\dagger} \Phi^{* 2} u^{2}} \frac{\tau^{* 1 / 2}}{\Phi^{*}} \sin \left(\frac{\Phi^{*} u}{\tau^{* 1 / 2}}\right) \sin \left(\Phi^{* 2} u\right) d u \\
& =\frac{2}{\pi} \int_{0}^{\infty} u^{-1} e^{-v^{\dagger} \Phi^{* 2} u^{2}} \sin \left(\Phi^{* 2} u\right) d u \\
& =\operatorname{erf}\left(v^{\dagger-1 / 2} \Phi^{*} / 2\right) \leq 1 \quad \text { from [20], }
\end{aligned}
$$

where $\Phi^{*}=\phi \tau^{*-1 / 2}$ is finite. Hence, when evaporation-condensation is present, the dimensionless rise approaches 2 . We comment that like the surface-diffusion only case, the function (C2) has the form of the self-similar solution for the development of the ramp modeled by the linear equation (4) when evaporation-condensation only is operative and $m \rightarrow \infty$ (Mullins [55]).

\section{References}

[1] G. L. J. Bailey and H. C. Watkins, "Surface tensions in the system solid copper-molten lead", Proc. Phys. Soc. B 63 (1950) 350-358.

[2] J. M. Blakely, Introduction to the Properties of Crystal Surfaces, Pergamon Press, Oxford (1973).

[3] G. W. Bluman and S. Kumei, "On the remarkable nonlinear diffusion equation $(\partial / \partial x)[a(u+$ $\left.b)^{-2}(\partial u / \partial x)\right]-(\partial u / \partial t)=0 "$, J. Math. Phys. 21 (1980) 1019-1023.

[4] G. W. Bluman and S. Kumei, Symmetries and Differential Equations Springer, New York (1989).

[5] H. P. Bonzel and E. Preuss, "Periodic surface profiles under the influence of anisotropic surface energy: a steady-state solution", Surface Sci. 145 (1984) 20-32.

[6] H. P. Bonzel, E. Preuss and B. Steffen, "The dynamical behaviour of periodic surface profiles on metals under the influence of anisotropic surface energy", Appl. Phys. A 35 (1984) 1-8.

[7] M. Bouchiba, "Sur l'équation de la corrosion intergranulaire par diffusion de surface", RAIRO Modél. Math. Anal. Numér. 21 (1987) 425-444.

[8] A. B. Brailsford and N. A. Gjostein, "Influence of the surface energy anisotropy on morphological changes occurring by surface diffusion", J. Appl. Phys. 46 (1975) 2390-2397.

[9] P. Broadbridge and I. White, "Constant rate rainfall infiltration: a versatile nonlinear model 1. analytic solution", Water Resour. Res. 24 (1988) 145-154.

[10] P. Broadbridge, J. H. Knight and C. Rogers, "Constant rate rainfall infiltration in a bounded profile: solutions of a nonlinear model", Soil Sci. Soc. Am. J. 52 (1988) 1526-1533.

[11] P. Broadbridge, "Exact solvability of the mullins nonlinear diffusion model of groove development", J. Math. Phys. 30 (1989) 1648-1651.

[12] P. Broadbridge and S. E. Godfrey, "Exact decaying soliton solutions of nonlinear Schrödinger equations: Lie-Bäcklund symmetries", J. Math. Phys. 32 (1991) 8-18.

[13] P. Broadbridge and C. Rogers, "On a nonlinear reaction-diffusion boundary value problem: application of a Lie-Bäcklund symmetry", J. Aust. Math. Soc. B 34 (1993) 318-332.

[14] P. Broadbridge and P. Tritscher, "An integrable fourth order nonlinear evolution equation applied to thermal grooving of metal surfaces", I.M.A. J. Appl. Math. 53 (1994) 249-265. 
[15] J. W. Cahn and J. E. Taylor, "Surface motion by surface diffusion", Acta Metall. Mater. 42 (1994) 1045-1063.

[16] J. Christian, The theory of transformations in metals and alloys, Pergamon, Oxford (1965).

[17] P. A. Clarkson, A. S. Fokas and M. J. Ablowitz, "Hodograph transformations of linearizable partial differential equations", SIAM J. Appl. Math. 49 (1989) 1188-1209.

[18] B. D. Coleman, R. S. Falk and M. Moakher, Rutgers Univ., unpublished research cited in J. W. Cahn and J. E. Taylor, "Surface motion by surface diffusion", Acta Metall. Mater. 42 (1994) 1045-1063 (p. 1047).

[19] F. Davi and M. E. Gurtin, "On the motion of a phase interface by surface diffusion", J. Appl. Math. Phys. (ZAMP) 41 (1990) 782-811.

[20] A. Erdelyi, W. Magnus, F. Oberhettinger and F. G. Tricomi, Higher transcendental functions, McGraw-Hill, New York (1953).

[21] H. E. Exner and E. Artz, "Sintering processes", in Physical Metallurgy (eds. R. W. Cahn and P. Haasen), Elsevier Science, New York (1983).

[22] A. S. Fokas, "A symmetry approach to exactly solvable evolution-equations", J. Math. Phys. 21 (1980) 1318-1325.

[23] H. Fujita, "The exact pattern of a concentration-dependent diffusion in a semi-infinite medium, part II", Text. Res. J. 22 (1952) 823-827.

[24] V. A. Galaktionov, V. A. Dorodnitsyn, G. G. Elenin, S. P. Kurdyumov and A. A. Samarskii, "A quasilinear heat equation with a source: peaking, localization, symmetry exact solutions, asymptotics, structures", J. Soviet Math. 41 (1988) 1222-1292.

[25] F. E. Génin, W. W. Mullins and P. Wynblatt, "The effect of stress on grain boundary grooving", Acta Metall. Mater. 41 (1993) 3541-3547.

[26] R. Ghez, “A generalized Gibbsian surface”, Surface Sci. 4 (1966) 125-140.

[27] N. A. Gjostein, "Adsorption and surface energy (II): thermal faceting from minimization of surface energy", Acta. Met. 11 (1963) 969-978.

[28] N. A. Gjostein, "Surface self diffusion of gold (I): Analysis of the scratch-flattening process", Trans. Metall. Soc. AIME 236 (1966) 1967-1977.

[29] E. E. Gruber and W. W. Mullins, "Extended analysis of surface scratch smoothing", Acta. Met. 14 (1966) 397-403.

[30] M. E. Gurtin, "On thermomechanical laws for the motion of a phase interface", J. Appl. Math. Phys. (ZAMP) 42 (1991) 370-388.

[31] S. A. Hackney and G. C. Ojard, "Grain boundary grooving at finite grain size", Scripta Met. 22 (1988) 1731-1735.

[32] C. Herring, "Effect of change of scale on sintering phenomena", J. Appl. Phys. 21 (1950) 301-303.

[33] C. Herring, "Surface tension as a motivation for sintering", in The Physics of Powder Metallurgy (ed. W. E. Kingston), McGraw-Hill, New York (1951).

[34] C. Herring, "The use of classical macroscopic concepts in surface-energy problems", in Structure and Properties of Solid Surfaces (eds. R. Gomer and C. S. Smith), Univ. of Chicago Press, Chicago (1952).

[35] J. M. Hill and V. G. Hart, "The Stefan problem in nonlinear heat conduction", J. Appl. Math. Phys. (ZAMP) 37 (1986) 206-229.

[36] J. E. Hilliard, M. Cohen and B. L. Averbach, "Grain-boundary energies in gold-copper alloys", Acta Met. 8 (1960) 26-31.

[37] H. Jones, P. H. Spriggs and G. M. Leak, "Relaxation of surface scratch by evaporation and condensation", Acta Met. 13 (1965) L444-446.

[38] Y. Karciga, Steep evaporating grooves, Honours thesis, Dept. of Mathematics, University of Wollongong, Australia, (1995).

[39] E. H. Kennard, Kinetic theory of gases, McGraw-Hill, New York (1938). 
[40] R. T. King and W. W. Mullins, "Theory of the decay of a surface scratch to flatness", Acta Met. 10 (1962) 601-606.

[41] A. Kitada, "On a property of a classical solution of the nonlinear mass-transport equation $u_{t}=$ $u_{x x} /\left(1+u_{x}^{2}\right) "$, J. Math. Phys. 27 (1986) 1391-1392.

[42] A. Kitada and H. Umehara, "On a property of a classical solution of the nonlinear mass-transport equation $u_{t}=u_{x x} /\left(1+u_{x}^{2}\right)$. II", J. Math. Phys. 28 (1987) 536-537.

[43] A. Kitada, "On a property of a classical solution of the nonlinear mass-transport equation $u_{\mathrm{t}}=$ $u_{x x} /\left(1+u_{x}^{2}\right)$. II: comment", J. Math. Phys. 28 (1987) 2982-2983.

[44] J. H. Knight, Solution of the nonlinear diffusion equation: existence, uniqueness and estimation, Ph. D. Thesis, Australian National University, Canberra (1973).

[45] J. H. Knight and J. R. Philip, "Exact solutions in nonlinear diffusion", J. Eng. Math. 8 (1974) 219-227.

[46] G. C. Kuczynski, "Self-diffusion in sintering of metallic particles", Trans. A.I.M.E. 185 (1949) 169-178.

[47] M. Z. C. Lee, A numerical model for dominant surface diffusion applied to periodic sputtering of surfaces, Honours thesis, Dept of Mathematics, University of Wollongong, Australia (1995).

[48] A. V. Mikhailov, A. B. Shabat and V. V. Sokolov, "The symmetry approach to classification of integrable equations", in What is integrability? (ed. V. E. Zakharov), Springer, Berlin (1990).

[49] A. J. W. Moore, "Thermal faceting", in Metal surfaces: structure, energetics and kinetics (eds. W. M. Robertson and N. A. Gjostein), ASM Metals Park, Ohio (1963).

[50] W. W. Mullins, "Theory of thermal grooving", J. Appl. Phys. 28 (1957) 333-339.

[51] W. W. Mullins, "The effect of thermal grooving on grain boundary motion", Acta Met. 6 (1958) 414-427.

[52] W. W. Mullins, "Flattening of a nearly plane solid surface due to capillarity", J. Appl. Phys. 30 (1959) 77-83.

[53] W. W. Mullins and P. G. Shewmon, "The kinetics of grain boundary grooving in copper", Acta Met. 7 (1959) 163-170.

[54] W. W. Mullins, "Theory of linear facet growth during thermal etching", Philos. Mag. 6 (1961) 1313-1341.

[55] W. W. Mullins, "Solid surface morphologies governed by capillarity", in Metal surfaces: structure, energetics and kinetics (eds. W. M. Robertson and N. A. Gjostein) ASM Metals Park, Ohio (1963).

[56] F. A. Nichols and W. W. Mullins, "Morphological changes of a surface of revolution due to capillarity-induced surface diffusion", J. Appl. Phys. 36 (1965) 1826-1835.

[57] T. P. Nolan and R. Sinclair, "Modeling of agglomeration in polycrystalline thin films: application to $\mathrm{TiSi}_{2}$ on a silicon substrate", J. Appl. Phys. 72 (1992) 720-724.

[58] P. J. Olver, "Evolution equations possessing infinitely many symmetries", J. Math. Phys. 18 (1977) 1212-1215.

[59] E. Preuss, N. Freyer and H. P. Bonzel, "Surface self-diffusion on Pt(110): directional dependence and influence of surface-energy anisotropy", Appl. Phys. A 41 (1986) 137-143.

[60] L. Ratke and H. J. Vogel, "Theory of grain boundary grooving in the convective-diffusive regime", Acta Metall. Mater. 39 (1991) 915-923.

[61] H. Riedel, Fracture at high temperatures Springer-Verlag, Berlin (1987).

[62] W. M. Robertson, "Grain-boundary grooving by surface diffusion for finite slopes", J. Appl. Phys. 42 (1971) 463-467.

[63] C. Rogers and W. M. Shadwick, Bäcklund transformations and their applications Academic Press, New York (1982).

[64] C. Rogers, M. P. Stallybrass and D. L. Clements, "On two phase filtration under gravity and with boundary infiltration: application of a Bäcklund transformation", Nonlin. Anal. Theory Methods Appl. 7 (1983) 785-799. 
[65] C. Rogers, "Application of rciprocal Bäcklund transformations to a class of nonlinear boundary value problems", J. Phys. A: Math. and Gen. 16 (1983) L493-495.

[66] C. Rogers, "Application of a reciprocal transformation to a two-phase Stefan Problem", J. Phys. A: Math. and Gen. 18 (1985) L105-109.

[67] C. Rogers, "On a class of moving boundary problems in non-linear heat conduction: application of a Bäcklund transformation", Int. J. Non-Linear Mech. 21 (1986) 249-256.

[68] G. C. Sander, J. Y. Parlange, V. Kühnel, W. L. Hogarth, D. Lockington and J. P. J. O'Kane, "Exact nonlinear solution for constant flux infiltration", J. Hydrol. (Neth.) 97 (1988) 341-346.

[69] S. R. Srinivasan and R. Trivedi, "Theory of grain boundary grooving under the combined action of the surface and volume diffusion mechanisms", Acta Metall. 21 (1973) 611-620.

[70] D. J. Srolovitz and S. A. Safran, "Capillary instabilities in thin films. I. energetics", J. Appl. Phys. 60 (1986) 247-254.

[71] D. J. Srolovitz and S. A. Safran, "Capillary instabilities in thin films. II. kinetics", J. Appl. Phys. 60 (1986) 255-260.

[72] M. L. Storm, "Heat conduction in simple metals", J. Appl. Phys. 22 (1951) 940-951.

[73] I. Sushumna and E. Ruckenstein, "The role of physical and chemical interactions in the behavior of supported metal-catalysts: iron on alumina, a case study", J. Catal. 94 (1985) 239-288.

[74] J. E. Taylor, J. W. Cahn and C. A. Handwerker, "I-Geometric models of crystal growth", Acta Metall. Mater. 40 (1992) 1443-1474.

[75] P. Tritscher, Integrable nonlinear evolution equations applied to solidification and surface redistribution, Ph. D. Thesis, University of Wollongong, Australia (1996).

[76] P. Tritscher and P. Broadbridge, "Grain boundary grooving by surface diffusion: an analytic nonlinear model for a symmetric groove", Proc. Roy. Soc. A (1995) 450, 569-587.

[77] H. J. Vogel and L. Ratke, "Instability of grain boundary grooves due to equilibrium grain boundary diffusion”, Acta Metall. Mater. 39 (1991) 641-649. 